\title{
MATERNAL OBESITY AND OBSTETRIC OUTCOIMES IN A TERTIARY CARE CENTER
}

KEY WORDS: Body Mass Index, gestational diabetes, preterm.

\section{Dr. H. Anitha Virgin Kumari*}

\section{Dr. S. Nivedita}

M.D,DGO, Associate Professor, Department Of Obstetrics And Gynecology, Govt.RSRM Lying In Hospital, Stanley Medical College, Chennai-13.

*Corresponding Author

MS OG,DNB OG, Assistant Professor, Department Of Obstetrics And Gynecology, Govt. RSRM Lying In Hospital, Stanley Medical College, Chennai-13.

BACKGROUND: Pregnancy is unique, yet normal physiological chapter in women's life. Pre-existing morbidity can complicate pregnancy affecting mother and fetus resulting in high risk pregnancy. One of which is obesity causing antepartum, intrapartum and postpartum complications both in mother and child. AIM OF THE STUDY: The aim of this study is to evaluate the effect of obesity on the maternal and perinatal outcome in pregnancies complicated by obesity. MATERIALS: A prospective study is done in Govt. RSRM lying in hospital during June 2019-june 2020 .Among antenatal mothers attending antenatal outpatient department, mothers were chosen in their first trimester who had Body Mass Index $>30 \mathrm{~kg} / \mathrm{m} 2$ as study group and mothers with a Body Mass Indexbetween $18.5 \mathrm{~kg} / \mathrm{m} 2 \mathrm{and} 25 \mathrm{~kg} / \mathrm{m} 2$ as control group. Detailed history taking and investigations done and they were followed up to delivery and postpartum until discharge and outcome studied. RESULTS: In the present study, increasing age, sedentary lifestyle and low socio economic status show a positive relation to obesity. The proportion of primiparous Women was more in obese group(51.9\%)when compared to control group(48.1\%). Higher incidence of gestational diabetes and pre eclampsia are seen in obese group. Higher rates of cesarean deliveries among this group. Preterm delivery in obese women $10.4 \%$ compared to control group. CONCLUSION: Maternal BMI has a strong association with pregnancy complications and outcome. The best time of intervention may be before a woman considers a pregnancy and attempts are to be made to maintain a normal BMI in women of childbearing age.

\section{INTRODUCTION}

The magnitude of the obesity prevalence has been increasing in developed and developing nations, though in varying degrees. It becomes a major issue when it affects the women of reproductive age group, by increasing the incidence of gestational diabetes, preeclampsia, gestational hypertension, labour induction, increased Caesarean rates, anesthetic complications, post-operative morbidity, prolonged hospital stay. They are at increased risk of delivering large babies and NICU admission. Although routine weighing of pregnant women is being carried out in all of the ante-natal clinics, not much of importance is given to the weight of the women as such. In fact pre-natal counseling plays a vital role in identifying women who are obese. Advice on weight reduction before embarking on pregnancy will go a long way in reducing the morbidity due to obesity in pregnancy.

\section{AIM OF THE STUDY}

The aim of this study is to evaluate the effect of obesity on the maternal and perinatal outcome in pregnancies complicated by obesity.

\section{MATERIALS AND METHODS}

MATERIALS: A prospective study is among antenatal women attending antenatal outpatient department at Govt. RSRM Lying in Hospital during the period of June 2019-June 2020. Cases were chosen such that their BMI during the first trimester is $>30 \mathrm{~kg} / \mathrm{m} 2$. Controls are antenatal women between $18.5 \mathrm{~kg} / \mathrm{m} 2$ and $25 \mathrm{~kg} / \mathrm{m} 2$ in first trimester.

METHODS: Pregnant mothers were selected according to the criteria and in all women detailed history followed by complete general and physical examination was done. Relevant hematological, biochemical investigations, USG were done. They were followed upto delivery and postpartum until discharge and outcome studied.

\section{RESULTS AND ANALYSIS}

TABLE 1: MATERNAL AGE DISTRIBUTION:

\begin{tabular}{|c|c|c|c|}
\hline \multirow[t]{2}{*}{ Maternal Age } & \multicolumn{2}{|c|}{ BMI } & \multirow[t]{2}{*}{ Total } \\
\hline & \begin{tabular}{|l|} 
control \\
\end{tabular} & Case & \\
\hline
\end{tabular}

\begin{tabular}{|c|c|c|c|c|c|}
\hline \multirow{12}{*}{$\begin{array}{l}\text { Age } \\
\text { Group in } \\
\text { years }\end{array}$} & \multirow[t]{3}{*}{$<20$} & Count & 5 & 2 & 7 \\
\hline & & \%within Age & $71.4 \%$ & $28.6 \%$ & $100.0 \%$ \\
\hline & & \%within BMI & $6.8 \%$ & $2.6 \%$ & $4.7 \%$ \\
\hline & \multirow[t]{3}{*}{$20-24$} & Count & 45 & 40 & 85 \\
\hline & & \%within Age & $52.9 \%$ & $47.1 \%$ & $100.0 \%$ \\
\hline & & \%within BMI & $61.6 \%$ & $51.9 \%$ & $56.7 \%$ \\
\hline & \multirow[t]{3}{*}{$24-29$} & Count & 17 & 20 & 37 \\
\hline & & \%within Age & $45.9 \%$ & $54.1 \%$ & $100.0 \%$ \\
\hline & & \%within BMI & $23.3 \%$ & $26.0 \%$ & $24.7 \%$ \\
\hline & \multirow[t]{3}{*}{$>30$} & Count & 6 & 15 & 21 \\
\hline & & \%within Age & $28.6 \%$ & $71.4 \%$ & $100.0 \%$ \\
\hline & & \%within BMI & $8.2 \%$ & $19.5 \%$ & $14.0 \%$ \\
\hline \multirow{3}{*}{\multicolumn{2}{|c|}{ Total }} & Count & 73 & 77 & 150 \\
\hline & & \%within Age & $48.7 \%$ & $51.3 \%$ & $100.0 \%$ \\
\hline & & \%within BMI & $100.0 \%$ & $100.0 \%$ & $100.0 \%$ \\
\hline
\end{tabular}

The majority of women in this study groupwere20-24years $(56.7 \%)$.The majority of obese women $(71.4 \%)$ were $>$ 30 years whereas majority of control women(71.4\%) were $<20 y r s$.

\section{TABLE 2: OBESITY AND PARITY}

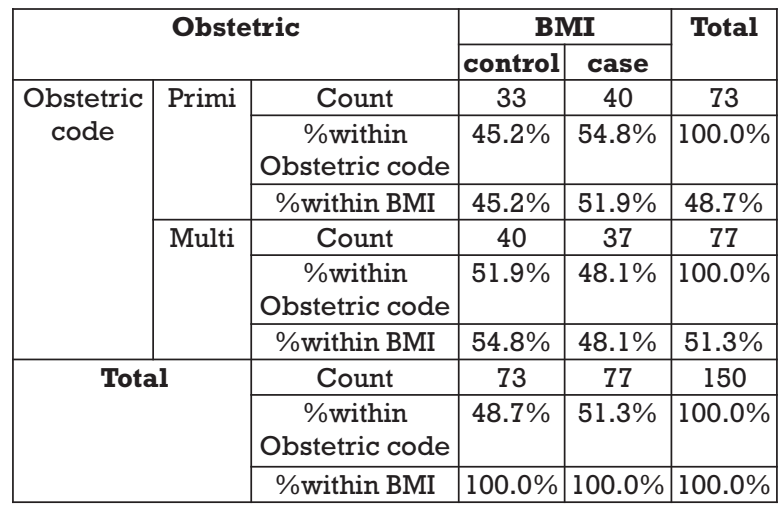

In this study it was observed that most of the primiparous patients were obese $51.9 \%$ whereas in multiparous $48.1 \%$. 
PARIPEX - INDIAN JOURNAL OF RESEARCH | Volume - 10 | Issue - 01 | January - 2021 | PRINT ISSN No. 2250 - 1991 | DOI : $10.36106 /$ paripex

\begin{tabular}{|c|c|c|c|c|c|}
\hline \multirow{2}{*}{\multicolumn{3}{|c|}{ Gestational age }} & \multicolumn{2}{|c|}{ BIMI } & \multirow{3}{*}{\begin{tabular}{|c|} 
Total \\
11 \\
\end{tabular}} \\
\hline & & & control & case & \\
\hline \multirow{9}{*}{$\begin{array}{c}\text { Gestational } \\
\text { age } \\
\text { (in weeks) }\end{array}$} & \multirow[t]{3}{*}{$<37$} & Count & 3 & 8 & \\
\hline & & $\begin{array}{c}\text { \%within } \\
\text { Gestational } \\
\text { age (in weeks) }\end{array}$ & $27.3 \%$ & $72.7 \%$ & $100.0 \%$ \\
\hline & & $\%$ within BMI & $4.1 \%$ & $10.4 \%$ & $7.3 \%$ \\
\hline & \multirow[t]{3}{*}{$37-40$} & Count & 56 & 56 & 112 \\
\hline & & $\begin{array}{c}\text { \%within } \\
\text { Gestational } \\
\text { age (in weeks) }\end{array}$ & $50.0 \%$ & $50.0 \%$ & $100.0 \%$ \\
\hline & & \% within BMI & $76.7 \%$ & $72.7 \%$ & $74.7 \%$ \\
\hline & \multirow[t]{3}{*}{$>40$} & Count & 14 & 13 & 27 \\
\hline & & $\begin{array}{c}\text { \%within } \\
\text { Gestational } \\
\text { age (in weeks) }\end{array}$ & $51.9 \%$ & $48.1 \%$ & $100.0 \%$ \\
\hline & & $\%$ within BMI & $19.2 \%$ & $16.9 \%$ & $18.0 \%$ \\
\hline \multirow{3}{*}{\multicolumn{2}{|c|}{ Total }} & Count & 73 & 77 & 150 \\
\hline & & $\begin{array}{c}\text { \%within } \\
\text { Gestational } \\
\text { age (in weeks) }\end{array}$ & $48.7 \%$ & $51.3 \%$ & $100.0 \%$ \\
\hline & & \% within BMI & $100.0 \%$ & $100.0 \%$ & $100.0 \%$ \\
\hline
\end{tabular}

\begin{tabular}{|c|c|c|c|c|}
\hline \multirow{3}{*}{$\begin{array}{c}\text { Repeat } \\
\text { Caeserean }\end{array}$} & Count & 9 & 17 & 26 \\
\hline & $\begin{array}{l}\text { \% within } \\
\text { Mode } \\
\text { of delivery }\end{array}$ & $34.6 \%$ & $65.4 \%$ & $100.0 \%$ \\
\hline & \%withinBMI & $12.3 \%$ & $22.1 \%$ & $17.3 \%$ \\
\hline \multirow[t]{3}{*}{ Forceps } & Count & 0 & 5 & 5 \\
\hline & $\begin{array}{l}\% \text { within } \\
\text { Mode } \\
\text { of delivery }\end{array}$ & $.0 \%$ & $100.0 \%$ & $100.0 \%$ \\
\hline & \%withinBMI & $.0 \%$ & $6.5 \%$ & $3.3 \%$ \\
\hline \multirow[t]{3}{*}{ Vaccum } & Count & 4 & 3 & 7 \\
\hline & $\begin{array}{l}\text { \% within } \\
\text { Mode } \\
\text { of delivery }\end{array}$ & $57.1 \%$ & $42.9 \%$ & $100.0 \%$ \\
\hline & \%withinBMI & $5.5 \%$ & $3.9 \%$ & $4.7 \%$ \\
\hline \multirow[t]{3}{*}{ VBAC } & Count & 1 & 0 & 1 \\
\hline & $\begin{array}{l}\% \text { within } \\
\text { Mode } \\
\text { of delivery }\end{array}$ & $100.0 \%$ & $.0 \%$ & $100.0 \%$ \\
\hline & \%within BMI & $1.4 \%$ & $.0 \%$ & $.7 \%$ \\
\hline \multirow[t]{3}{*}{ Total } & Count & 73 & 77 & 150 \\
\hline & $\begin{array}{l}\% \text { within } \\
\text { Mode } \\
\text { of delivery }\end{array}$ & $48.7 \%$ & $51.3 \%$ & $100.0 \%$ \\
\hline & \%within BMI & $100.0 \%$ & $100.0 \%$ & $100.0 \%$ \\
\hline
\end{tabular}

Majority of women in our study group delivered at term. Preterm delivery in obese women is $10.4 \%$ compared to control group $4.1 \%$.

\section{TABLE 4: ANTEPARTUM COMPLICATIONS}

\begin{tabular}{|c|c|c|c|c|}
\hline \multicolumn{2}{|c|}{ Antepartum Complication } & \multicolumn{2}{|c|}{ BMI } & \multirow{3}{*}{$\begin{array}{c}\text { Total } \\
16 \\
\end{array}$} \\
\hline & & Control & case & \\
\hline \multirow{3}{*}{ GHTN } & Count & 0 & 16 & \\
\hline & $\begin{array}{c}\% \text { within Antepartum } \\
\text { Complication }\end{array}$ & $.0 \%$ & $100.0 \%$ & $100.0 \%$ \\
\hline & \%within BMI & $.0 \%$ & $20.8 \%$ & $10.7 \%$ \\
\hline \multirow{3}{*}{$\begin{array}{c}\text { Severe } \\
\text { Pre } \\
\text { Eclampsia }\end{array}$} & Count & 1 & 3 & 4 \\
\hline & $\begin{array}{c}\% \text { within Antepartum } \\
\text { Complication }\end{array}$ & $25.0 \%$ & $75.0 \%$ & $100.0 \%$ \\
\hline & \%within BMI & $1.4 \%$ & $3.9 \%$ & $2.7 \%$ \\
\hline \multirow{3}{*}{ GDM } & Count & 0 & 8 & 8 \\
\hline & $\begin{array}{c}\% \text { within Antepartum } \\
\text { Complication }\end{array}$ & $.0 \%$ & $100.0 \%$ & $100.0 \%$ \\
\hline & \%within BMI & $.0 \%$ & $10.4 \%$ & $5.3 \%$ \\
\hline \multirow{3}{*}{ Anaemia } & Count & 9 & 4 & 13 \\
\hline & $\begin{array}{c}\% \text { within Antepartum } \\
\text { Complication }\end{array}$ & $69.2 \%$ & $30.8 \%$ & $100.0 \%$ \\
\hline & \%within BMI & $12.3 \%$ & $5.2 \%$ & $8.7 \%$ \\
\hline \multirow[t]{3}{*}{ Total } & Count & 73 & 77 & 150 \\
\hline & $\begin{array}{c}\% \text { within Antepartum } \\
\text { Complication }\end{array}$ & $48.7 \%$ & $51.3 \%$ & $100.0 \%$ \\
\hline & \%within BMI & $100.0 \%$ & $100.0 \%$ & $100.0 \%$ \\
\hline
\end{tabular}

The incidence of gestational diabetes was $10.4 \% \%$ and $.0 \%$ respectively in obese and control group. The incidence of preeclampsia was $3.9 \%$ and $1.4 \%$ in obese and control group respectively. The incidence of gestational hypertension was $20.8 \%$ and $.0 \%$ in obese and control group. The results were statistically significant.

\section{TABLE 5: Mode of Delivery}

\begin{tabular}{|c|c|c|c|c|c|}
\hline \multicolumn{3}{|c|}{ Mode of Delivery } & \multicolumn{2}{|c|}{ BIMI } & \multirow{2}{*}{ Total } \\
\cline { 3 - 5 } \multirow{2}{*}{\begin{tabular}{c} 
Mode $\begin{array}{c}\text { of } \\
\text { delivery }\end{array}$ \\
\cline { 3 - 5 }
\end{tabular}} & $\begin{array}{c}\text { Labour } \\
\text { natural }\end{array}$ & Count & 46 & 20 & 66 \\
\cline { 3 - 5 } & $\begin{array}{c}\text { \% within } \\
\text { mode } \\
\text { of delivery }\end{array}$ & $69.7 \%$ & $30.3 \%$ & $100.0 \%$ \\
\cline { 3 - 5 } & \%withinBMI & $63.0 \%$ & $26.0 \%$ & $44.0 \%$ \\
\cline { 3 - 5 } & $\begin{array}{c}\text { Primary } \\
\text { Caeserean }\end{array}$ & $\begin{array}{c}\text { Count } \\
\text { \% within } \\
\text { Mode } \\
\text { of delivery }\end{array}$ & $28.9 \%$ & $71.1 \%$ & $100.0 \%$ \\
\cline { 3 - 5 } & $\%$ withinBMI & $17.8 \%$ & $41.6 \%$ & $30.0 \%$ \\
\hline
\end{tabular}

Among obese women group $41.6 \%$ delivery by primary Caesarean.

In normal BMI 63\% delivered by labour natural. Repeat Caesarean rate was also higher. Five delivered by Outlet forceps .Vacuum delivery higher in case of control group $5.5 \%$ than in obese 3.9\%. Among the indication for Primary Caesarean section Failed Induction highest with $4.7 \%$

\section{TABLE 6: Intrapartum Complication}

\begin{tabular}{|c|c|c|c|c|}
\hline \multicolumn{2}{|c|}{ Intrapartum Complication } & \multicolumn{2}{|c|}{ BMI } & \multirow{3}{*}{$\begin{array}{c}\text { Total } \\
1\end{array}$} \\
\hline & & control & case & \\
\hline \multirow{3}{*}{$\begin{array}{l}\text { Shoulder } \\
\text { Dystocia }\end{array}$} & Count & 0 & 1 & \\
\hline & $\begin{array}{c}\text { \%within Intrapartum } \\
\text { Complication }\end{array}$ & $.0 \%$ & $100.0 \%$ & $100.0 \%$ \\
\hline & \%withinBMI & $.0 \%$ & $1.4 \%$ & $.7 \%$ \\
\hline \multirow[t]{3}{*}{ Increase BP } & Count & 2 & 1 & 3 \\
\hline & $\begin{array}{r}\% \text { within Intrap } \\
\text { Complicati }\end{array}$ & $66.7 \%$ & $33.3 \%$ & 100.0 \\
\hline & \%withinBMI & $2.7 \%$ & $1.3 \%$ & $2.0 \%$ \\
\hline \multirow{3}{*}{$\begin{array}{l}\text { Abruptio } \\
\text { placenta }\end{array}$} & Count & 0 & 2 & 2 \\
\hline & $\begin{array}{c}\text { \%within Intrapartum } \\
\text { Complication }\end{array}$ & $.0 \%$ & $100.0 \%$ & $100.0 \%$ \\
\hline & \%withinBMI & $.0 \%$ & $2.6 \%$ & $1.3 \%$ \\
\hline \multirow[t]{3}{*}{ Total } & Count & 73 & 77 & 150 \\
\hline & $\begin{array}{c}\text { \%within Intrapartum } \\
\text { Complication }\end{array}$ & $48.7 \%$ & $51.3 \%$ & $100.0 \%$ \\
\hline & \%withinBMI & $100.0 \%$ & $100.0 \%$ & $100.0 \%$ \\
\hline
\end{tabular}

\section{TABLE 7: POSTPARTUM COMPLICATION}

\begin{tabular}{|c|c|c|c|c|}
\hline \multicolumn{2}{|c|}{ Postpartum Complication } & \multicolumn{2}{|c|}{ BMI } & \multirow{2}{*}{ Total } \\
\cline { 2 - 5 } Control & case & \\
\hline \multirow{3}{*}{$\begin{array}{c}\text { Wound } \\
\text { infection }\end{array}$} & Count & 0 & 5 & 5 \\
\cline { 2 - 5 } & $\begin{array}{c}\text { \% within Postpartum } \\
\text { Complication }\end{array}$ & $.0 \%$ & $100.0 \%$ & $100.0 \%$ \\
\cline { 2 - 5 } & $\%$ withinBMI & $.0 \%$ & $6.5 \%$ & $3.3 \%$ \\
\hline Anemia & Count & 4 & 1 & 5 \\
\cline { 2 - 5 } & $\begin{array}{c}\text { \% within Postpartum } \\
\text { Complication }\end{array}$ & $80.0 \%$ & $20.0 \%$ & $100.0 \%$ \\
\cline { 2 - 5 } & \%withinBMI & $5.5 \%$ & $1.3 \%$ & $3.3 \%$ \\
\hline \multirow{2}{*}{ GHTN } & Count & 0 & 1 & 1 \\
\cline { 2 - 5 } & $\begin{array}{c}\text { \% within Postpartum } \\
\text { Complication }\end{array}$ & $.0 \%$ & $100.0 \%$ & $100.0 \%$ \\
\cline { 2 - 5 } & $\%$ withinBMI & $.0 \%$ & $1.3 \%$ & $.7 \%$ \\
\hline
\end{tabular}




\begin{tabular}{|c|c|c|c|c|}
\hline HELLP/AKI & Count & 0 & 1 & 1 \\
\cline { 2 - 5 } & $\begin{array}{c}\% \text { within Postpartum } \\
\text { Complication }\end{array}$ & $.0 \%$ & $100.0 \%$ & $100.0 \%$ \\
\cline { 2 - 5 } & \%withinBMI & $.0 \%$ & $1.3 \%$ & $.7 \%$ \\
\hline AtonicPPH & Count & 0 & 2 & 2 \\
\cline { 2 - 5 } & $\begin{array}{c}\text { \% within Postpartum } \\
\text { complications }\end{array}$ & $.0 \%$ & $100.0 \%$ & $100.0 \%$ \\
\cline { 2 - 5 } Fever & \%withinBMI & $.0 \%$ & $2.6 \%$ & $1.3 \%$ \\
\cline { 2 - 5 } & $\begin{array}{c}\text { Count } \\
\text { within Postpartum } \\
\text { Complication }\end{array}$ & $.0 \%$ & $100.0 \%$ & $100.0 \%$ \\
\cline { 2 - 5 } & \%withinBMI & $.0 \%$ & $1.3 \%$ & $.7 \%$ \\
\hline \multirow{2}{*}{ Total } & Count & 73 & 77 & 150 \\
\cline { 2 - 5 } & $\begin{array}{c}\text { within Postpartum } \\
\text { Complication }\end{array}$ & $48.7 \%$ & $51.3 \%$ & $100.0 \%$ \\
\cline { 2 - 5 } & \%withinBMI & $100.0 \%$ & $100.0 \%$ & $100.0 \%$ \\
\hline
\end{tabular}

Five obese patients developed wound infection, in control group no wound infection was found. One obese patient with elevated blood pressure and one obese patient with HELLP/AKI who recovered Anemia rate in control group was higher $(5.5 \%)$ and lower in obese group(1.3\%). Deep vein thrombosis was not seen in either group.

$93.5 \%$ of control women delivered at term and $6.5 \%$ of obese women and $4.1 \%$ of control group delivered preterm.

\section{TABLE 8: INDICATIONS FOR NICU ADIMISSION}

\begin{tabular}{|c|c|c|c|c|c|}
\hline \multirow{2}{*}{\multicolumn{3}{|c|}{\begin{tabular}{|c|} 
INDICATION FOR NICU \\
ADIMISSION
\end{tabular}}} & \multicolumn{2}{|c|}{ BIMI } & \multirow{3}{*}{$\begin{array}{r}\text { Total } \\
14 \\
\end{array}$} \\
\hline & & & \multirow{2}{*}{$\frac{\text { control }}{7}$} & \multirow{2}{*}{\begin{tabular}{|c|} 
Case \\
7
\end{tabular}} & \\
\hline \multirow{21}{*}{$\begin{array}{l}\text { admission } \\
\text { and } \\
\text { indication }\end{array}$} & \multirow[t]{3}{*}{$\mathrm{NNH}$} & Count & & & \\
\hline & & $\begin{array}{c}\% \text { within } \\
\text { Neonatal } \\
\text { morbidity/ } \\
\text { mortality }\end{array}$ & $50.0 \%$ & $50.0 \%$ & $\begin{array}{c}100.0 \\
\%\end{array}$ \\
\hline & & \%within BMI & $33.3 \%$ & $25.0 \%$ & $28.6 \%$ \\
\hline & \multirow[t]{3}{*}{ Preterm } & Count & 0 & 1 & 1 \\
\hline & & $\begin{array}{c}\% \text { within } \\
\text { Neonatal } \\
\text { morbidity/ } \\
\text { mortality }\end{array}$ & $.0 \%$ & $\begin{array}{c}100.0 \\
\%\end{array}$ & $\begin{array}{c}100.0 \\
\%\end{array}$ \\
\hline & & \%within BMI & $.0 \%$ & $3.6 \%$ & $2.0 \%$ \\
\hline & \multirow[t]{3}{*}{ Fever } & Count & 0 & 1 & 1 \\
\hline & & $\begin{array}{c}\% \text { within } \\
\text { Neonatal } \\
\text { morbidity/ } \\
\text { mortality }\end{array}$ & $.0 \%$ & $\begin{array}{c}100.0 \\
\%\end{array}$ & $\begin{array}{c}100.0 \\
\%\end{array}$ \\
\hline & & \%within BMI & $.0 \%$ & $3.6 \%$ & $2.0 \%$ \\
\hline & \multirow[t]{3}{*}{ IDM } & Count & 1 & 4 & 5 \\
\hline & & $\begin{array}{c}\% \text { within } \\
\text { Neonatal } \\
\text { morbidity/ } \\
\text { mortality }\end{array}$ & $20.0 \%$ & $80.0 \%$ & $\begin{array}{c}100.0 \\
\%\end{array}$ \\
\hline & & \%within BMI & $4.8 \%$ & $14.3 \%$ & $10.2 \%$ \\
\hline & \multirow{3}{*}{$\begin{array}{l}\text { Respiratory } \\
\text { distress }\end{array}$} & Count & 2 & 7 & 9 \\
\hline & & $\begin{array}{c}\% \text { within } \\
\text { Neonatal } \\
\text { morbidity/ } \\
\text { mortality }\end{array}$ & $22.2 \%$ & $77.8 \%$ & $\begin{array}{c}100.0 \\
\%\end{array}$ \\
\hline & & $\%$ within BMI & $9.5 \%$ & $25.0 \%$ & $18.4 \%$ \\
\hline & \multirow[t]{3}{*}{ LBW } & Count & 5 & 1 & 6 \\
\hline & & $\begin{array}{c}\% \text { within } \\
\text { Neonatal } \\
\text { morbidity/ } \\
\text { mortality }\end{array}$ & $83.3 \%$ & $16.7 \%$ & $\begin{array}{c}100.0 \\
\%\end{array}$ \\
\hline & & \%within BMI & $23.8 \%$ & $3.6 \%$ & $12.2 \%$ \\
\hline & \multirow{3}{*}{$\begin{array}{c}\text { Birth } \\
\text { Asphyxia }\end{array}$} & Count & 2 & 1 & 3 \\
\hline & & $\begin{array}{c}\% \text { within } \\
\text { Neonatal } \\
\text { morbidity/ } \\
\text { mortality }\end{array}$ & $66.7 \%$ & $33.3 \%$ & $\begin{array}{c}100.0 \\
\%\end{array}$ \\
\hline & & \%within BMI & $9.5 \%$ & $3.6 \%$ & $6.1 \%$ \\
\hline
\end{tabular}

\begin{tabular}{|c|c|c|c|c|}
\hline MSAF & Count & 1 & 1 & 2 \\
\cline { 2 - 5 } & $\begin{array}{c}\% \text { within } \\
\text { Neonatal } \\
\text { morbidity/ } \\
\text { mortality }\end{array}$ & $50.0 \%$ & $50.0 \%$ & 100.0 \\
$\%$ & & & \\
\cline { 2 - 5 } & $\%$ within BMI & $4.8 \%$ & $3.6 \%$ & $4.1 \%$ \\
\hline Macrosomia & Count & 0 & 1 & 1 \\
\cline { 2 - 5 } & $\begin{array}{c}\% \text { within } \\
\text { Neonatal } \\
\text { morbidity/ } \\
\text { mortality }\end{array}$ & $.0 \%$ & $\begin{array}{c}100.0 \\
\%\end{array}$ & $\begin{array}{c}100.0 \\
\%\end{array}$ \\
\cline { 2 - 6 } & $\%$ within BMI & $.0 \%$ & $3.6 \%$ & $2.0 \%$ \\
\hline
\end{tabular}

Neonates of obese mothers had increased NICU admission. $18.4 \%$ of babies born to obese women and $13.4 \%$ babies of control women were admitted in and in control group in this study was due to Neonatal Hyperbilirubinaemia; for obese group maximum was due to respiratory distress(25\%).

\section{CONCLUSION}

Our study highlights the importance of obesity as a public health issues. The numerous maternal and perinatal risks in obese pregnant women which pose a considerable challenge to the obstetrical practitioner. Maternal BMI has a strong association with pregnancy complications and outcome. The best time of intervention may be before a woman considers a pregnancy and attempts are to be made to maintain a normal BMI in women of childbearing age. Prepregnancy counselling, health programs and appropriate multidisciplinary management should be done.

Obesity not only impacts the health of the women but also child leading to childhood obesity and diabetes. Pregnancies among obese women must be classified as high risk pregnancies and appropriate antenatal care should be provided with heightened surveillance, anticipation and diagnosis of the complications and intervene earlier, if complications arise.

\section{REFERENCES}

1. Park's TextBook of Preventive and Social Medicine.

2. High riskPregnancy:Management options:D.K.James

3. American college of Obstetric and Gynaecologists.FAQ182,April2016

4. Bautista Castafio2013:Maternal obesity in Early pregnancy and risk of adverse outcomes

5. BaetenJM,BukusiEA,LambeM.Pregnancy complications and outcomes among overweight and obese nulliparous women. Am J PubHealth.2001 91:436-440.

6. CraneSS,WojtwoyczMA, DyeTD,etal. Association between pre- pregnancy obesity and the risk of cesarean delivery. ObestetGynecol. 1997:89:213-216.

7. EdwardsLE,DickesWF,AltonIR, etal.Pregnancy complications and birth outcomes in the massively obese;course, outcome, and obesity prognosis of the infant. Am JObstetGynecol. 1978;131:479-48

8. Galtiere-DereureF,MontpeyrouxF,BoulotP,etal.Weight excess before pregnancy:complications and cost.IntJobes. 1995:19:443-448.

9. GarbaciakJA, Richter M, Miller S, et al Maternal Weight and pregnancy complications AmJ ObstetGynecol. 1985:152:238-245.

10. Galtiere-DereureF,MontpeyrouxF,BoulotP,etal. Weight excess before pregnancy: complications and cost.IntJobes.1995:19:443-448.

11. GarbaciakJA, Richter M, Miller S, et al Maternal Weight and pregnancy complications AmJ ObstetGynecol. 1985:152:238-245.

12. Meaghan Aleddy,MichaelLPower,JaySchulkin:The Impact of Maternal Obesity and Fetal Health.

13. Pallavi Singh,RekhaWadhwani, Maternal and fetal outcome in High BMI.

14. GRamoniene2017.Maternal obesity and obstetrics outcome in tertiary health care. 